\title{
Toxoplasma gondii infection and chronic schizophrenia: is there any association?
}

\author{
Salvina Maria de Campos-Carli ${ }^{1}$, Érica Leandro Marciano Vieira ${ }^{1}$, Natalia Pessoa Rocha ${ }^{1,2}$, Keliane de Oliveira ${ }^{3}$, \\ Fernanda Carneiro Guimarães 3 , Izabela Guimarães Barbosa ${ }^{1}$, João Luís Vieira Monteiro de Barrosi, Olaoluwa \\ Okusaga², Olindo Assis Martins-Filho ${ }^{4}$, João Vinícius Salgado ${ }^{1,3}$, Antônio Lúcio Teixeira1,2 \\ 1 Interdisciplinary Laboratory of Medical Research, Faculty of Medicine, Federal University of Minas Gerais (UFMG), Belo Horizonte, MG, Brazil. \\ 2 Neuropsychiatry Program, Department of Psychiatry and Behavioral Sciences, McGovern Medical School, The University of Texas Health Science Center at Houston, Houston, TX, USA. \\ 3 Instituto Raul Soares, Hospital Foundation of the State of Minas Gerais (FHEMIG), Belo Horizonte, MG, Brazil. \\ ${ }^{4}$ Laboratory of Diagnostic and Monitoring Biomarkers, René Rachou Research Center, Oswaldo Cruz Foundation (Fiocruz), Belo Horizonte, MG, Brazil.
}

Received: 03/07/2017 - Accepted: 10/14/2017

DOI: 10.1590/0101-60830000000140

\section{ABSTRACT}

Background: Toxoplasma gondii (T. gondii) infection has been identified as a risk factor for schizophrenia. Objectives: Herein, we sought to evaluate the association between T. gondii infection and clinical symptoms and quality of life in patients with schizophrenia. Methods: We conducted a cross-sectional study with 48 patients with chronic schizophrenia and 40 controls. Peripheral blood was drawn, and IgM and IgG anti-T. gondii antibodies were evaluated by Enzyme-Linked Immunosorbent Assay (ELISA). Depressive, positive and negative symptoms were assessed, respectively, by the Calgary Depression Scale (CDS) and the Positive and Negative Syndrome Scale (PANSS). Cognitive performance was assessed in patients by the Brazilian version of the Schizophrenia Cognition Rating Scale (SCoRS-BR). Quality of life was assessed by the Brazilian version of the Quality of Life in Schizophrenia scale (QLS-BR). Results: The prevalence and titers of $T$. gondii IgM and IgG antibodies did not differ between patients and controls. The positive serology for T. gondii IgG antibodies was not associated with illness symptoms, cognitive performance, depressive symptoms or quality of life. Discussion: Our findings suggest that toxoplasmosis infection is not associated with severity of symptoms, quality of life, cognitive or depressive symptoms in schizophrenia patients.

Campos-Carli SM et al. / Arch Clin Psychiatry. 2017;44(6):145-8

Keywords: Toxoplasma gondii, schizophrenia, cognition, depression, quality of life.

\section{Introduction}

Schizophrenia is a pervasive psychiatry disease whose etiopathogenesis results from complex gene-environmental interactions. Accumulating evidence suggests that infection is an important environmental factor implicated in schizophrenia pathogenesis ${ }^{1}$. Epidemiological and meta-analytic studies have shown an association between infections and increased risk of schizophrenia ${ }^{2}$.

Toxoplasma gondii is a protozoan with a worldwide distribution ${ }^{3}$. It has been estimated that up to one third of the world population has been exposed to the parasite 4 . The exposure rate and the prevalence of T. gondii infection varies according to geographic region, population habits as well as socioeconomic conditions ${ }^{5}$. For instance, in Brazil the rate of T. gondii infection in humans is high: up to $50 \%$ of elementary school children and $50-80 \%$ of women of child-bearing age have antibodies to T. gondiit.

In healthy adults, infection with $T$. gondii generally results in a latent infection that persists for life. This chronic infection was largely regarded as asymptomatic, but this assumption has changed lately. Latent toxoplasmosis has been linked to changes in cognitive function in both children ${ }^{7}$ and elderly individuals ${ }^{8}$. T. gondii infection has also been regarded as a risk factor for schizophrenia. A recent metaanalysis showed that T. gondii infection is associated with several psychiatric disorders including schizophrenia, bipolar disorder (BD) and obsessive-compulsive disorder ${ }^{9}$. Noteworthy, the reactivation of latent $T$. gondii infection may occur in patients with schizophrenia ${ }^{9}$. Moreover, T. gondii infection was associated with an increased risk for mortality in patients with schizophrenia ${ }^{10}$.

Although the association between T. gondii infection and schizophrenia has already been investigated, the results are still controversial and only few studies have focused on whether the infection is associated with clinical outcomes. Therefore, this study was carried out to investigate differences in T. gondii seroprevalence in patients with schizophrenia and controls, as well as to evaluate whether seroprevalence and anti- $T$. gondii antibody titers are associated with clinical parameters.

\section{Methods}

\section{Subjects}

This is a cross-sectional study in which 48 patients with chronic schizophrenia and 40 controls were evaluated. Schizophrenia diagnosis was confirmed by the structured psychiatric interview MINI-Plus ${ }^{11}$ using the DSM-IV-TR criteria ${ }^{12}$.

Inclusion criteria for patients were: (a) age between 18-60 years; (b) diagnosis of schizophrenia; c) patients who were clinically stable as assessed by scores on the PANSS (see below). Controls were recruited from the local community using the following inclusion criteria (a) age between 18-60 years; (b) no current or past history of psychiatric disorder; (c) no family history of major psychiatry disorder. Exclusion criteria for both groups were: (a) history of epilepsy, dementia, brain trauma, tumor or other primary neurological diseases; (b) current dependence/use of drugs (alcohol, cannabis, cocaine, opioid); (c) current or recent (past four weeks) infections; (d) autoimmune or severe clinical diseases; (e) current or recent (past four weeks) use of corticosteroid or anti-inflammatory drugs. The study conformed to the declaration of Helsinki, with its protocol approved by the local Ethics Research Committee. Both patients and controls provided written informed consent.

\section{Clinical, cognitive and quality of life assessments}

Positive and negative symptoms were assessed by the Positive and Negative Syndrome Scale, PANSS13. Clinical stability was defined as 
a PANSS positive score of 19 or less, and less than 4 in any individual item of the positive scale for at least four weeks. The clinical stability criterion was based on positive symptoms due to their high impact on cognitive functioning assessment. The validity and applicability of SCoRS has been demonstrated in patients with clinical stable schizophrenia, but not in patients with a recent exacerbation of the illness and/or with significant positive symptomatology ${ }^{14}$. The Calgary Depression Scale (CDS) was used to assess depressive symptoms ${ }^{15}$.

Cognitive performance was assessed by the Brazilian version of the Schizophrenia Cognition Rating Scale (SCoRS-BR) ${ }^{16}$. This is an interview-based scale that includes information provided by both the patient and an informant. It generates three different ratings: from the patient, from the informant and from the interviewer. The interview is composed of 18 items covering several cognitive domains, including memory, attention, reasoning and problem solving, working memory, motor skills and language. The total score is the sum of scores on the 18 SCoRS items with a higher the score indicating greater degree of cognitive impairment. A global rating is also generated ${ }^{17}$.

Quality of life was assessed by the Quality of Life in Schizophrenia Scale (QLS) validated for use in Brazil (QLS-BR) ${ }^{18}$. This scale has a total of 21 items distributed among three factors: i) instrumental domain, ii) intrapsychic foundations and iii) interpersonal relations domain. Items include information on patient functioning during the three weeks preceding the interview. The sum of the values obtained in the 21 items of the scale is used to assess the overall quality of life. Higher scores denote better quality of life.

\section{Serological evaluation}

Peripheral blood was drawn from each subject by venipuncture into a vacuum tube without anticoagulant. Blood was immediately centrifuged at 3,000 rpm for 10 minutes, serum was collected and kept frozen at $-80^{\circ} \mathrm{C}$ until assayed. IgM and IgG anti-T. gondii antibodies were determined by Enzyme-Linked Immunosorbent Assay (ELISA), using kits SERION - ELISA classic test by VIRION (Würzburg, Germany), following the manufacturer's guidelines. The test sensitivity were $10 \mathrm{IU} / \mathrm{mL}$ and $5 \mathrm{IU} / \mathrm{mL}$ to $\mathrm{IgM}$ and $\mathrm{IgG}$, respectively.

\section{Statistical analysis}

All variables were tested for Gaussian distribution by the KolmogorovSmirnov normality test. Association between dichotomous variables was assessed with the Fisher's exact test. Two groups were compared by Mann-Whitney or Student's $t$ tests when non-normally or normally distributed, respectively. Spearman's correlation analyses were performed for correlation analyses. All statistical tests were two-tailed and were performed using a significance level of $\alpha=0.05$. Statistical analyses were performed using SPSS software version 22.0 (SPSS Inc., Chicago, IL, USA), as well as GraphPad Prism 5.0 (GraphPad Software, Inc., La Jolla, California, EUA).

\section{Results}

\section{Demographic and clinical data}

Demographic and clinical data are shown in Table 1. Patients and controls did not differ regarding age and gender. Patients with schizophrenia had less years of formal education than controls. The mean $( \pm$ SD) length of illness was $15.20( \pm 10.50)$ years. The mean $( \pm$ SD) scores in the positive and negative subscales of the PANSS were, respectively, $10.28( \pm 2.78)$ and $19.67( \pm 7.73)$, indicating a chronic condition with predominance of negative symptoms.

Table 1. Demographic, clinical and serological features of controls and patients

\begin{tabular}{|c|c|c|c|}
\hline & Controls $(\mathrm{N}=40$ ) & Patients SZ (N = 48) & $p$ values \\
\hline Male gender [frequency in \% (N)] & $42.50(17)$ & $56.25(27)$ & $0.284 a$ \\
\hline Age in years (mean $\pm S D$ ) & $40.62 \pm 10.29$ & $40.21 \pm 9.59$ & $0.845^{b}$ \\
\hline Educational levels in years (mean $\pm S D$ ) & $12.25 \pm 4.77$ & $8.04 \pm 4.25$ & $<0.001^{c}$ \\
\hline Age of onset in years (mean \pm SD) & - & $24.58 \pm 9.91$ & - \\
\hline Length of illness in years (mean $\pm S D$ ) & _ & $15.20 \pm 10.50$ & _ \\
\hline \multicolumn{4}{|l|}{ Medication in use [frequency in \% (N)] } \\
\hline Potent typical antipsychotics & _ & $60.86(28)$ & _- \\
\hline Sedatives typical antipsychotics & __ & $39.13(18)$ & _ \\
\hline Atypical antipsychotics & __ & $34.78(16)$ & _- \\
\hline Clozapine & __ & $4.34(2)$ & _ \\
\hline Mood stabilizers & - & $8.69(4)$ & _- \\
\hline Antidepressants & _- & $6.52(3)$ & _ \\
\hline Chlorpromazine equivalent (mean \pm SD) & _- & $351.74 \pm 232.29$ & _- \\
\hline Preview electroconvulsive therapy [frequency in \% (N)] & _ & $8.33(4)$ & _ \\
\hline \multicolumn{4}{|l|}{ PANSS (mean \pm SD) } \\
\hline Positive & _- & $10.28 \pm 2.78$ & _- \\
\hline Negative & _- & $19.67 \pm 7.73$ & _- \\
\hline General & _- & $26.22 \pm 7.27$ & _- \\
\hline Total & _- & $56.55 \pm 12.07$ & _- \\
\hline CDS (mean \pm SD) & _- & $1.95 \pm 2.54$ & _- \\
\hline \multicolumn{4}{|l|}{ SCoRS-BR (mean \pm SD) } \\
\hline SCoRS-BR patient & _- & $37.48 \pm 11.12$ & __ \\
\hline SCoRS-BR informant & _- & $36.59 \pm 10.40$ & _- \\
\hline SCoRS-BR interviewer & _- & $40.49 \pm 11.38$ & _- \\
\hline SCoRS-BR global & & $5.64 \pm 1.58$ & \\
\hline QLS-BR total /126 (mean $\pm S D$ ) & & $54.41 \pm 24.76$ & _- \\
\hline Positive IgM antiboties anti-T. gondii (\%) (N) & $7.50(3)$ & $4.17(2)$ & $0.656 \mathrm{a}$ \\
\hline Positive lgG antibodies anti-T. gondii (\%) (N) & $56.41(22)^{d}$ & $56.25(27)$ & $1.000 \mathrm{a}$ \\
\hline $\operatorname{lgM} \mathrm{IU} / \mathrm{mL}$ (mean \pm SD) & $130.67 \pm 114.82$ & $121.31 \pm 97.99$ & $0.630 \mathrm{c}$ \\
\hline $\lg G \mathrm{IU} / \mathrm{mL}($ mean \pm SD) & $115.59 \pm 166.53$ & $127.58 \pm 218.12$ & $0.785^{\circ}$ \\
\hline
\end{tabular}

a Fisher's exact test; ${ }^{b}$ Student's t test; $\mathrm{c}$ Mann-Whitney test; ${ }^{\circ}$ One individual presented indeterminate results for lgG.

CDS: Calgary Depression Scale; PANSS: Positive and Negative Syndrome Scale; QLS-BR: Quality of Life in Schizophrenia scale validated for use in Brazil; SCoRS-BR: Brazilian version of the Schizophrenia Cognition Rating Scale; SD: standard deviation. 


\section{T. gondii seroprevalence and association with clinical parameters}

Patients with schizophrenia and controls presented similar seroprevalence and titers for both IgG and IgM anti-T. gondii antibodies (Table 1).

Patients with schizophrenia were then categorized into two groups: anti-T gondii IgG positive (Toxoplasma-seropositive, $\mathrm{N}=27$ ) and anti-T gondii IgG negative (Toxoplasma-seronegative, $\mathrm{N}=21$ ). We did not find any difference between Toxoplasma-seronegative and Toxoplasma-seropositive patients regarding age, gender, educational level, age of onset, length of illness, chlorpromazine equivalent level and PANSS subsets (Table 2). Toxoplasma-seropositive and Toxoplasma-seronegative patients presented similar scores in the scales that evaluated depressive symptoms, cognitive performance and quality of life (Table 2).

We did not find any significant correlation between anti- $T$. gondii IgM and IgG levels and age, age at onset, duration of illness, chlorpromazine equivalent level, positive PANSS, negative PANSS, total PANSS, patient SCoRS, informant SCoRS, interviewer SCoRS, global SCoRS, CDS or QLS scores. No difference was found when comparing male and female participants.

\section{Discussion}

The current study was conducted in order to compare the prevalence of $T$. gondii infection between patients with schizophrenia and controls, as well as to evaluate the potential association between T. gondii infection and clinical symptoms in Brazilian patients with schizophrenia. We found similar rates of positive serological reaction to $T$. gondii in controls and patients with schizophrenia. In addition, we did not find any association between $T$. gondii seroprevalence and demographic data, positive, negative and depressive symptoms, cognitive performance and quality of life in patients with schizophrenia.

Previous studies found higher rates of T. gondii infection in patients with schizophrenia in comparison with controls ${ }^{19-23}$. In contrast, some authors have questioned this association ${ }^{24,25}$. A recent meta-analysis reported a significant association between T. gondii infection as estimated by IgG antibodies and schizophrenia (odds ratio $1.81, \mathrm{P}<0.0001)$. A very high heterogeneity was observed among studies, and the amplitude of the odds ratio was influenced by region and general seroprevalence ${ }^{9}$. Accordingly, the possibility of finding association between toxoplasmosis and schizophrenia is lower in regions with high seroprevalence rates when compared with regions with low rates. Brazil has a high prevalence of T. gondii infection which could be an explanation for the current findings. Moreover, our sample was composed by chronic patients under high dose of antipsychotics that may affect anti-T. gondii IgG titers ${ }^{26}$.

T. gondii infection has been associated with symptom severity in schizophrenia ${ }^{23,27,28}$. In addition, latent toxoplasmosis may have a negative impact on the disease course and treatment response in patients with schizophrenia ${ }^{29}$. Corroborating these clinical data, patients with schizophrenia $T$. gondii-seropositive presented more significant morphological changes in the caudate, cingulate, thalamus and occipital cortex in comparison with seronegative patients ${ }^{30}$. In the current study, we did not find significant differences between seropositive and seronegative patients regarding the clinical symptoms of schizophrenia as assessed by PANSS. The fact that the enrolled patients were clinically stable comprising a relatively homogenous sample may partially explain this result.

Data on whether T. gondii infection is associated with cognitive aspects in schizophrenia are still controversial. For instance, one study showed that people diagnosed with schizophrenia who were exposed in utero to T. gondii presented impaired performance in two executive function tests: the Wisconsin Card Sorting Test (WCST) and the Trail Making Test (TMT) part B ${ }^{31}$. These patients also exhibited deficits on figural fluency, letter-number sequencing and backward digit span ${ }^{31}$. Conversely, other studies failed to show any association between latent toxoplasmosis and cognitive function in schizophrenia ${ }^{32,33}$. According to our results, T. gondii infection is not associated with self-reported cognitive performance as evaluated by the SCoRS. Discrepancies among studies can also be explained, at least in part, by strain related specific effects of T. gondii on human brain 9 . Indeed, regional differences in strain distribution have been reported, and $T$. gondii strain-specific effects in depression have already been demonstrated 9 .

It is worth mentioning that the use of antipsychotic and/or mood stabilizers presenting in vitro anti-toxoplasmic activity was associated with a better clinical outcome in a population of BD patients. Patients with BD seropositive for T. gondii presented more lifetime depressive episodes when treated by drugs having no anti-toxoplasmic activity

Table 2. Demographic, clinical, cognitive performance and quality of life in patients with schizophrenia, according to serology for lgG anti-T.gondii

\begin{tabular}{|c|c|c|c|}
\hline & Seronegative $(\mathrm{N}=21)$ & Seropositive ( $\mathrm{N}=27$ ) & $p$ value \\
\hline Male gender [frequency in \% (N)] & $66.67(14)$ & $48.15(13)$ & $0.249 \mathrm{a}$ \\
\hline Age in years (mean $\pm S D$ ) & $39.71 \pm 10.10$ & $40.59 \pm 9.35$ & $0.757^{b}$ \\
\hline Educational level in years (mean \pm SD) & $8.10 \pm 4.81$ & $8.00 \pm 3.86$ & $0.940^{\mathrm{b}}$ \\
\hline Age of onset in years (mean $\pm S D$ ) & $23.25 \pm 9.81$ & $25.61 \pm 10.04$ & $0.428^{b}$ \\
\hline Length of illness in years (mean $\pm S D$ ) & $15.94 \pm 11.19$ & $14.69 \pm 10.37$ & $0.715^{b}$ \\
\hline Chlorpromazine equivalent (mean $\pm S D$ ) & $375.78 \pm 233.31$ & $333.71 \pm 234.49$ & $0.637 \mathrm{c}$ \\
\hline \multicolumn{4}{|l|}{ PANSS (mean \pm SD) } \\
\hline Positive & $10.67 \pm 2.82$ & $9.96 \pm 2.76$ & $0.306^{c}$ \\
\hline Negative & $21.24 \pm 7.97$ & $18.36 \pm 7.41$ & $0.212^{\mathrm{b}}$ \\
\hline General & $28.05 \pm 6.49$ & $24.76 \pm 7.66$ & $0.133^{\mathrm{b}}$ \\
\hline Total & $58.50 \pm 11.75$ & $54.77 \pm 12.35$ & $0.324 \mathrm{~b}$ \\
\hline $\mathrm{CDS}($ mean $\pm \mathrm{SD})$ & $1.81 \pm 2.31$ & $2.04 \pm 2.72$ & $0.777 \mathrm{c}$ \\
\hline \multicolumn{4}{|l|}{ SCoRS-BR (mean \pm SD) } \\
\hline SCoRS-BR patient & $35.70 \pm 9.19$ & $38.85 \pm 12.40$ & $0.431 \mathrm{c}$ \\
\hline SCoRS-BR informant & $32.65 \pm 6.57$ & $39.64 \pm 11.86$ & $0.072 \mathrm{c}$ \\
\hline SCoRS-BR interviewer & $36.71 \pm 7.65$ & $43.41 \pm 13.01$ & $0.124 c$ \\
\hline SCoRS-BR global & $5.27 \pm 1.07$ & $5.95 \pm 1.88$ & $0.591^{b}$ \\
\hline QLS-BR total/126 (mean $\pm S D$ ) & $60.65 \pm 25.76$ & $49.10 \pm 23.20$ & $0.160^{\mathrm{b}}$ \\
\hline
\end{tabular}

a Fisher's exact test; ${ }^{\mathrm{b}}$ Student's t test; c Mann-Whitney test.

CDS: Calgary Depression Scale; PANSS: Positive and Negative Syndrome Scale; OLS-BR: Quality of Life in Schizophrenia scale validated for use in Brazil; SCoRS-BR: Brazilian version of the Schizophrenia Cognition Rating Scale; SD: standard deviation. 
(e.g. aripiprazole, carbamazepine, clozapine, lamotrigine, lithium, olanzapine, quetiapine) compared to patients who received drugs with anti-toxoplasmic activity (e.g. fluphenazine, haloperidol, levomepromazine, paliperidone, risperidone, thioridazine, valproate) ${ }^{34}$. It remains to be determined whether the treatment with drugs with anti-toxoplasmic activity is also associated with better clinical outcomes of the patients with schizophrenia.

We are aware of the limitations of our study. First the sample size is small and consists of chronic patients using antipsychotics. It is uncertain whether our results can be applied to patients with prodromal or early schizophrenia. Cohort studies involving a larger sample of drug naïve patients are critical to confirm the specificity to clarify the pathogenic role of T. gondii in schizophrenia and its symptoms. Cognitive assessment of patients was based on self-reported measures. Although SCoRS is highly correlated to cognitive performance and functioning, a comprehensive neuropsychological evaluation in both patients and controls could add more specific information, including the domains potentially affected by toxoplasmosis.

In conclusion, our data showed that $T$. gondii infection is not associated with schizophrenia. In addition, T. gondii seroprevalence and levels of IgG antibodies were not associated with demographic data, disease severity, depressive and cognitive symptoms and quality of life. Our results might be influenced by strain-specific brain effects and high prevalence of T. gondii infection in our sample. Further studies are needed in order to elucidate the role of $T$. gondii in schizophrenia pathophysiology.

\section{References}

1. Brown AS. Prenatal infection as a risk factor for schizophrenia. Schizophr Bull. 2006;32(2):200-2.

2. Arias I, Sorlozano A, Villegas E, de Dios Luna J, McKenney K, Cervilla $\mathrm{J}$, et al. Infectious agents associated with schizophrenia: a meta-analysis. Schizophr Res. 2012;136(1-3):128-36.

3. Hill DE, Chirukandoth S, Dubey JP. Biology and epidemiology of Toxoplasma gondii in man and animals. Anim Health Res Rev. 2005;6(1):41-61.

4. Tenter AM, Heckeroth AR, Weiss LM. Toxoplasma gondii: from animals to humans. Int J Parasitol. 2000;30(12-13):1217-58.

5. Montoya JG, Liesenfeld O. Toxoplasmosis. Lancet. 2004;363(9425): 1965-76.

6. Dubey JP, Lago EG, Gennari SM, Su C, Jones JL. Toxoplasmosis in humans and animals in Brazil: high prevalence, high burden of disease, and epidemiology. Parasitology. 2012;139(11):1375-424.

7. Mendy A, Vieira ER, Albatineh AN, Gasana J. Toxoplasma gondii seropositivity and cognitive functions in school-aged children. Parasitology. 2015;142(9):1221-7.

8. Nimgaonkar VL, Yolken RH, Wang T, Chang CC, McClain L, McDade E, et al. Temporal Cognitive Decline Associated With Exposure to Infectious Agents in a Population-based, Aging Cohort. Alzheimer Dis Assoc Disord. 2016;30(3):216-22.

9. Sutterland AL, Fond G, Kuin A, Koeter MW, Lutter R, van Gool T, et al. Beyond the association. Toxoplasma gondii in schizophrenia, bipolar disorder, and addiction: systematic review and meta-analysis. Acta Psychiatr Scand. 2015;132(3):161-79.

10. Dickerson F, Boronow J, Stallings C, Origoni A, Yolken R. Toxoplasma gondii in individuals with schizophrenia: association with clinical and demographic factors and with mortality. Schizophr Bull. 2007;33(3): 737-40.

11. Amorim P, Lecrubier Y, Weiller E, Hergueta T, Sheehan D. DSM-IH-R Psychotic Disorders: procedural validity of the Mini International Neuropsychiatric Interview (MINI). Concordance and causes for discordance with the CIDI. Eur Psychiatry. 1998;13(1):26-34.

12. Association AP. Diagnostic and Statistical Manual of Mental Disorders, Fourth Edition, Text Revision (DSM-IV TR). Washington, DC: American Psychiatric Association; 2000.

13. Kay SR, Fiszbein A, Opler LA. The positive and negative syndrome scale (PANSS) for schizophrenia. Schizophr Bull. 1987;13(2):261-76.

14. Vita A, Deste G, Barlati S, De Peri L, Giambra A, Poli R, et al. Interviewbased assessment of cognition in schizophrenia: applicability of the
Schizophrenia Cognition Rating Scale (SCoRS) in different phases of illness and settings of care. Schizophr Res. 2013;146(1-3):217-23.

15. Bressan RA, Chaves AC, Shirakawa I, de Mari J. Validity study of the Brazilian version of the Calgary Depression Scale for Schizophrenia. Schizophr Res. 1998;32(1):41-9.

16. Ferreira Junior BC, Barbosa MA, Barbosa IG, Borges A, Hara C, Rocha FL Versão brasileira da Escala de Avaliação da Cognição em Esquizofrenia (SCoRSBr) - Validação em contextos clínicos sem informantes. J Bras Psiquiatr. 2010;59(4):271-8.

17. Keefe RS, Poe M, Walker TM, Kang JW, Harvey PD. The Schizophrenia Cognition Rating Scale: an interview-based assessment and its relationship to cognition, real-world functioning, and functional capacity. Am J Psychiatry. 2006;163(3):426-32.

18. Cardoso CS, Bandeira M, Caiaffa WT, Fonseca JOP. Escala de qualidade de vida para pacientes com esquizofrenia (QLS-BR): adaptação transcultural para o Brasil. J Bras Psiquiatr. 2002;51:31-8.

19. Alvarado-Esquivel C, Estrada-Martinez S, Liesenfeld O. Toxoplasma gondii infection in workers occupationally exposed to unwashed raw fruits and vegetables: a case control seroprevalence study. Parasit Vectors. 2011;4:235.

20. Hinze-Selch D, Daubener W, Eggert L, Erdag S, Stoltenberg R, Wilms S. A controlled prospective study of toxoplasma gondii infection in individuals with schizophrenia: beyond seroprevalence. Schizophr Bull. 2007;33(3):782-8.

21. Niebuhr DW, Millikan AM, Cowan DN, Yolken R, Li Y, Weber NS. Selected infectious agents and risk of schizophrenia among U.S. military personnel. Am J Psychiatry. 2008;165(1):99-106.

22. Torrey EF, Bartko JJ, Yolken RH. Toxoplasma gondii and other risk factors for schizophrenia: an update. Schizophr Bull. 2012;38(3):642-7.

23. Wang HL, Wang GH, Li QY, Shu C, Jiang MS, Guo Y. Prevalence of Toxoplasma infection in first-episode schizophrenia and comparison between Toxoplasma-seropositive and Toxoplasma-seronegative schizophrenia. Acta Psychiatr Scand. 2006;114(1):40-8.

24. Emelia O, Amal RN, Ruzanna ZZ, Shahida H, Azzubair Z, Tan KS, et al. Seroprevalence of anti-Toxoplasma gondii IgG antibody in patients with schizophrenia. Trop Biomed. 2012;29(1):151-9.

25. Karabulut N, Bilgic S, Gurok MG, Karaboga F. Is there any role of latent toxoplasmosis in schizophrenia disease? J Chin Med Assoc. 2015;78(9):533-7.

26. Webster JP, Lamberton PH, Donnelly CA, Torrey EF. Parasites as causative agents of human affective disorders? The impact of anti-psychotic, moodstabilizer and anti-parasite medication on Toxoplasma gondii's ability to alter host behaviour. Proc Biol Sci. 2006;273(1589):1023-30.

27. Yolken RH, Dickerson FB, Fuller Torrey E. Toxoplasma and schizophrenia. Parasite Immunol. 2009;31(11):706-15.

28. Holub D, Flegr J, Dragomirecka E, Rodriguez M, Preiss M, Novak T, et al. Differences in onset of disease and severity of psychopathology between toxoplasmosis-related and toxoplasmosis-unrelated schizophrenia. Acta Psychiatr Scand. 2013;127(3):227-38.

29. Celik T, Kartalci S, Aytas O, Akarsu GA, Gozukara H, Unal S. Association between latent toxoplasmosis and clinical course of schizophrenia continuous course of the disease is characteristic for Toxoplasma gondiiinfected patients. Folia Parasitol (Praha). 2015;62.

30. Horacek J, Flegr J, Tintera J, Verebova K, Spaniel F, Novak T, et al Latent toxoplasmosis reduces gray matter density in schizophrenia but not in controls: voxel-based-morphometry (VBM) study. World J Biol Psychiatry. 2012;13(7):501-9.

31. Brown AS, Vinogradov S, Kremen WS, Poole JH, Deicken RF, Penner JD et al. Prenatal exposure to maternal infection and executive dysfunction in adult schizophrenia. Am J Psychiatry. 2009;166(6):683-90.

32. Shirts BH, Prasad KM, Pogue-Geile MF, Dickerson F, Yolken RH, Nimgaonkar VL. Antibodies to cytomegalovirus and Herpes Simplex Virus 1 associated with cognitive function in schizophrenia. Schizophr Res. 2008;106(2-3):268-74.

33. Yolken RH, Torrey EF, Lieberman JA, Yang S, Dickerson FB. Serological evidence of exposure to Herpes Simplex Virus type 1 is associated with cognitive deficits in the CATIE schizophrenia sample. Schizophr Res. 2011;128(1-3):61-5.

34. Fond G, Boyer L, Gaman A, Laouamri H, Attiba D, Richard JR, et al Treatment with anti-toxoplasmic activity (TATA) for toxoplasma positive patients with bipolar disorders or schizophrenia: a cross-sectional study J Psychiatr Res. 2015;63:58-64. 